\title{
ProBDNF Signaling Regulates Depression-Like Behaviors in Rodents under Chronic Stress
}

\author{
Yin-Yin Bai ${ }^{1,2,7}$, Chun-Sheng Ruan ${ }^{1,3,7}$, Chun-Rui Yang ${ }^{1,7}$, Jia-Yi Li ${ }^{3}$, Zhi-Long Kang', Li Zhou', Dennis Liu ${ }^{4}$, \\ Yue-Qing Zeng' ${ }^{1}$, Ting-Hua Wang ${ }^{2}$, Chang-Fu Tian ${ }^{5}$, Hong Liao ${ }^{6}$, Larisa Bobrovskaya ${ }^{3}$ and Xin-Fu Zhou*,1,3 \\ 'Key Laboratory of Stem Cell and Regenerative Medicine, Institute of Molecular and Clinical Medicine, Kunming Medical University, Kunming, \\ China; ${ }^{2}$ Institute of Neuroscience, Molecular Clinic Institute, Kunming Medical University, Kunming, China; ${ }^{3}$ School of Pharmacy and Medical \\ Sciences, Division of Health Sciences, University of South Australia, Adelaide, SA, Australia; ${ }^{4}$ Discipline of Psychiatry, School of Medicine, University of \\ Adelaide, Adelaide, SA, Australia; ${ }^{5}$ Biomedical Engineering Research Center, Kunming Medical University, Kunming, China; ${ }^{6}$ Jiangsu Key Laboratory \\ of Drug Screening, China Pharmaceutical University, Nanjing, China
}

\begin{abstract}
Chronic exposure to stressful environment is a key risk factor contributing to the development of depression. However, the mechanisms involved in this process are still unclear. Brain-derived neurotropic factor (BDNF) has long been investigated for its positive role in regulation of mood, although the role of its precursor, proBDNF, in regulation of mood is not known. In this study, using an unpredictable chronic mild stress (UCMS) paradigm we found that the protein levels of proBDNF were increased in the neocortex and hippocampus of stressed mice and this UCMS-induced upregulation of proBDNF was abolished by chronic administration of fluoxetine. We then established a rat model of UCMS and found that the expression of proBDNF/p $75^{\mathrm{NTR}} / \mathrm{sortilin}$ was upregulated, whereas the expression of mature BDNF and TrkB was downregulated in both neocortex and hippocampus of chronically stressed rats. Finally, we found that the injection of anti-proBDNF antibody via intracerebroventricular (i.c.v.) and intraperitoneal (i.p.) approaches into the UCMS rats significantly reversed the stress-induced depression-like behavior and restored the exploratory activity and spine growth. Although intramuscular injection of AAV-proBDNF did not exacerbate the UCMS-elicited rat mood-related behavioral or pathological abnormalities, i.c.v. injection of AAV-proBDNF increased the depression-like behavior in naive rats. Our findings suggest that proBDNF plays a role in the development of chronic stress-induced mood disturbances in rodents. Central (i.c.v.) or peripheral (i.p.) inhibition of proBDNF by injecting specific antiproBDNF antibodies may provide a novel therapeutic approach for the treatment of stress-related mood disorders.
\end{abstract}

Neuropsychopharmacology (2016) 4I, 2882-2892; doi:I 0.1038/npp.2016.100; published online I3 July 2016

\section{INTRODUCTION}

Depression is a highly prevalent mood disorder that affects the life quality of $17 \%$ of population during lifetime. Genetic and environmental factors play critical roles in the development of this disorder. Failure to cope with the stressful life events has long been recognized as a most common risk in the development of depression (Olff et al, 2005). To date, there is no available medication that can alleviate all the symptoms. Current antidepressants can only be effective in a certain population and the therapeutic effect appears 2 weeks after the treatment with antidepressants. Thus, understanding the etiological mechanisms of depression is essential for seeking novel, more effective medication options.

*Correspondence: Professor X-F Zhou, School of Pharmacy and Medical Sciences, Division of Health Sciences, University of South Australia, Adelaide, SA 5000, Australia, Tel: +61 88302 2329, Fax: +6I

88302 1087, E-mail: xin-fu.zhou@unisa.edu.au

${ }^{7}$ These three authors contributed equally to this work.

Received 10 January 20 I6; revised 9 June 20 16; accepted I2 June 20 I6; accepted article preview online 17 June 2016
Brain-derived neurotropic factor (BDNF), the most widely distributed neurotrophin in the central nervous system, functions to facilitate the neuronal survival, migration, differentiation, neurite growth, and synaptic plasticity (Huang and Reichardt, 2001; Lewin and Barde, 1996) and has long been implicated in mood regulation by enhancing such neuronal activities. In as early as 1995, Duman's group (Nibuya et al, 1995) found that antidepressants and related medications increased the protein levels of BDNF and its receptor TrkB in rat brains. Furthermore, Siuciak et al (1997) have shown that administration of BDNF in rat midbrain can reverse depression-like behavior. A clinical study in 2004 has shown that serum BDNF levels negatively correlated with the depression-related personality traits (Lang et al, 2004). Afterwards, serum and plasma BDNF levels have been found decreased in patients with major depressive disorder (MDD) (Lee et al, 2007; Piccinni et al, 2008). These clinical studies have suggested that peripheral BDNF levels can serve as a biomarker for depression. However, the link between central and peripheral BDNF in mood regulation is still unclear. In 2010, Schmidt and Duman (2010) showed that peripheral administration of BDNF decreased basal depression- and 
anxiety-like behaviors as well as chronic unpredictable stress-induced depression-like behavior in mice; the peripheral administration of BDNF also increased the adult hippocampal neurogenesis and led to increased levels of central BDNF and its signaling, suggesting that peripherally administered BDNF can reach the brain. These findings also suggest that both central and peripheral BDNF can regulate mood performance.

As the precursor form of BDNF, proBDNF has been found to play an opposing role to BDNF in regulating neuronal activity (Sun et al, 2012; Woo et al, 2005; Xu et al, 2011). It is well established that BDNF regulates neuronal survival via activation of a membrane receptor, TrkB, whereas proBDNF regulates neuronal death via activating membrane receptor p75 ${ }^{\mathrm{NTR}}$-mediated apoptotic signaling that requires another membrane receptor called sortilin (Lee et al, 2001; Nykjaer et al, 2004; Teng et al, 2005). Our previous clinical study has shown that the blood proBDNF levels as well as its co-receptors, $\mathrm{p} 75^{\mathrm{NTR}}$ and sortilin, are increased in the patients with MDD (Zhou et al, 2013). However, the role of proBDNF and its signaling in the central nervous system in mood regulation remains unknown.

In this study, we have investigated the role of proBDNF in a chronic stress model of depression in rodents and found that proBDNF may be involved in the pathogenesis of this disorder.

\section{MATERIALS AND METHODS}

\section{Animals}

Male, 2-3-month-old C57BL/6 mice (25-30 g) and Wistar rats (200-250 g) were purchased from the Vital River Laboratories (Beijing, China) (Supplementary Figures S1-S3), and Wistar rats (200-250 g) in Figure 6 were purchased through the Reid Animal Facility (RAF; University of South Australia, UniSA). All animals were housed in the animal house of the Kunming Medical University (or RAF) with free access to water and food. All experimental procedures involving animals were approved by the Department of Laboratory Animal Sciences of Kunming Medical University (or SA Pathology Animal Ethics Committee, UniSA), and conducted between 0700 and $1900 \mathrm{~h}$ in accordance with the National Institutes of Health Guide for the Care and Use of Laboratory Animals (or the National Health and Medical Research Council of Australia). As this is an exploratory study, we initially designed this study to minimize the group variation by recruiting a single sexual population in rodents. Males were chosen because of potential hormonal influences on mood regulation in females (progesterone) (Schechter, 1998; Sherwin, 1991) and because of the differential regulation of BDNF in male and female rodents (Autry et al, 2009; Chourbaji et al, 2011, 2012).

\section{Unpredictable Chronic Mild Stress (UCMS) Paradigm}

The UCMS paradigm was conducted as previously described (Yang et al, 2014). Briefly, animals for UCMS were singly housed and daily experienced a random stimulus for the below 12 stressors for 28 days in mice or 21 days in rats (see Supplementary Figure S1): (1) cage shaking (1 time/s, $5 \mathrm{~min})$; (2) cage tilting $45(8 \mathrm{~h})$; (3) cold swim $\left(13 \pm 1{ }^{\circ} \mathrm{C}\right.$,
$5 \mathrm{~min}$ ); (4) food and water deprivation (24h); (5) tail pinching (60 s); (6) moist bedding ( $8 \mathrm{~h})$; (7) warm swim $\left(37 \pm 2{ }^{\circ} \mathrm{C}, 5 \mathrm{~min}\right)$; (8) overnight illumination (12 h); (9) tail pinching (90 s); (10) no stress; (11) reversing day and night (24 h); and (12) tail pinching (120 s). The control animals were socially housed (5 animals/cage) and left undisturbed unless necessary procedures including routine cleaning. At the end of the UCMS protocol, open field (mouse, day 29; rat, day 22), forced swimming (mouse, day 30; rat, day 23), and sucrose preference tests (mouse, day 31; rat, day 24) were performed to assess mood-related behaviors of UCMS animals.

\section{Drug Administration}

The clinical antidepressant, fluoxetine (H20110442, Lilly, Fegersheim, France) (Ruan et al, 2014, 2015), was employed to daily treat the UCMS mice during the UCMS protocol via intraperitoneal (i.p.) injection $(20 \mathrm{mg} / \mathrm{kg}$, dissolved in sterilized saline).

\section{Animal Surgery and Microinjection}

On completion of the UCMS protocol, the UCMS rats were subjected to different treatments as follows: (1) anesthetized with pentobarbital sodium and placed in a stereotaxic apparatus and intracerebroventricularly (i.c.v.) injected with one single dose of sheep anti-proBDNF (Fan et al, 2008; Lim et al, 2015) antibody $(10 \mu \mathrm{g}, 1 \mu \mathrm{g} / \mu \mathrm{l})$ on day 22 with a Hamilton syringe, and then behaviorally assessed on days 23, 24, and 25 (Figure 3a); (2) i.p. injected with one dose of anti-proBDNF antibody $(10 \mu \mathrm{l}, 0.08 \mu \mathrm{g} / \mu \mathrm{l}$ per $\mathrm{g}$ of body weight) on day 22 , followed by one half the dose on day 26 , and behaviorally assessed on days 27, 28, and 29 (Figure 3a); and (3) i.m. injected with one dose of AAV-proBDNF or AAV-BDNF vectors $\left(50 \mu \mathrm{l}, 0.5 \times 10^{9} \mathrm{VG} / \mu \mathrm{l}\right.$; Virovek, Hayward, CA) in tibialis anterior muscle on day 22, and behaviorally assessed on days 37, 38, and 39 (Figure 4a). The same volume of normal sheep serum (i.c.v. and i.p. injections) or AAV-EGFP (i.m. injection) was injected to rats (as a negative control). In a separate experiment, naive rats (not exposed to UCMS) were randomly grouped for behavioral testing on days 1 (open field test) and 2 (forced swimming test) (Figure 6a), and received one dose of i.c.v. injection of AAV-proBDNF or AAV-EGFP $(4 \mu \mathrm{l}$, $\left.0.5 \times 10^{9} \mathrm{VG} / \mu \mathrm{l}\right)$ on day 3 as described above. After 15 days, behavioral tests were performed on days 19 and 20. On day 21, brain tissues were harvested for ELISA assay and immunostaining analysis.

\section{Behavioral Testing}

Open field, forced swimming, and sucrose preference tests were performed as previously described (Yang et al, 2014). Detailed procedures are described in Supplementary Material.

\section{mRNA and Protein Detections}

Western blot, RT-PCR, ELISA, and immunostaining were performed as previously described (Fan et al, 2008; Lim et al, 
2015; Yang et al, 2014), and detailed procedures are described in Supplementary Material.

\section{Golgi Staining}

Animals were humanely killed and brain tissues were isolated for Golgi staining with FD Rapid Golgi-Stain Kit (FD Neurotechnologies, Columbia, MD) according to the manufacturer's instructions. After staining, brains were cut into $150 \mu \mathrm{m}$ coronal sections with Leica cryostat (CM3050S, Germany). Sections were mounted on gelatine-coated slides and allowed to dry overnight at room temperature, shielded from light. The sections were then stained, dehydrated, and mounted. Five random visual fields in the neocortex or hippocampus were selected, and the secondary branches of dendrites were chosen for imaging using Leica TCS SP5X confocal microscope; the mean length of spines from each field was analyzed by Leica LAS AF Lite software.

\section{Data Analysis}

All data are presented as mean \pm SEM and analyzed by IBM SPSS Statistics 21. For difference comparison within groups, Student's $t$-test was used for two groups, one-way ANOVA for more than two groups, and two-way ANOVA for two groups with two factors. The $p<0.05$ was considered statistically significant.

\section{RESULTS}

\section{Chronic Stress Increased proBDNF Levels in Mouse Brains}

Clinical studies have shown that serum levels of proBDNF are increased in patients with MDD (Zhou et al, 2013); however, whether chronic stress can regulate proBDNF levels in the brain is still unknown. In this study, we have used an UCMS model of mice to examine the expression profile of proBDNF in the brain. This model is well established in our laboratory and we have previously shown that UCMS consistently induces depression-like behaviors in mice (Ruan et al, 2014, 2015; Yang et al, 2014, 2015). After 28 days of UCMS protocol, the proBDNF levels were significantly increased in neocortex (Student's $t$-test, $t_{(10)}=-4.17$, $p=0.014$; Supplementary Figures S2B and C) and hippocampus (Student's $t$-test, $t_{(10)}=-2.64, p=0.048$; Supplementary Figures S2D and E) of stressed mice $(n=6)$ vs control mice $(n=6)$.

\section{Fluoxetine Abolished the Increase in proBDNF Levels in the Brain of Mice Exposed to Chronic Stress}

To examine whether upregulation of proBDNF caused by chronic stress is reversible, the selective serotonin reuptake inhibitor antidepressant fluoxetine was used to chronically treat the UCMS mice. At the end of treatment protocol, behavioral assessments and analysis of proBDNF in the brain were conducted in all three groups of animals (control, UCMS, and UCMS+fluoxetine; $n=12$ each). We have found that chronic stress significantly increased the immobility in UCMS mice $v s$ control mice in the open field test, and fluoxetine significantly reversed this behavior (one-way a

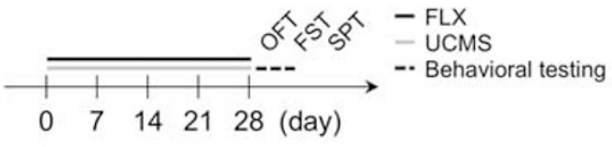

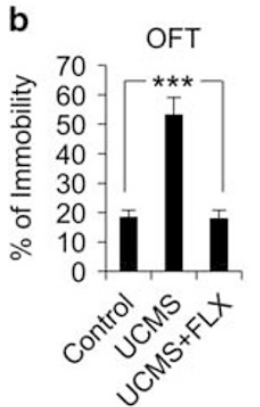

e
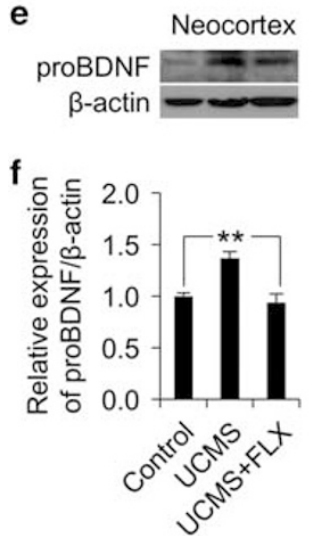
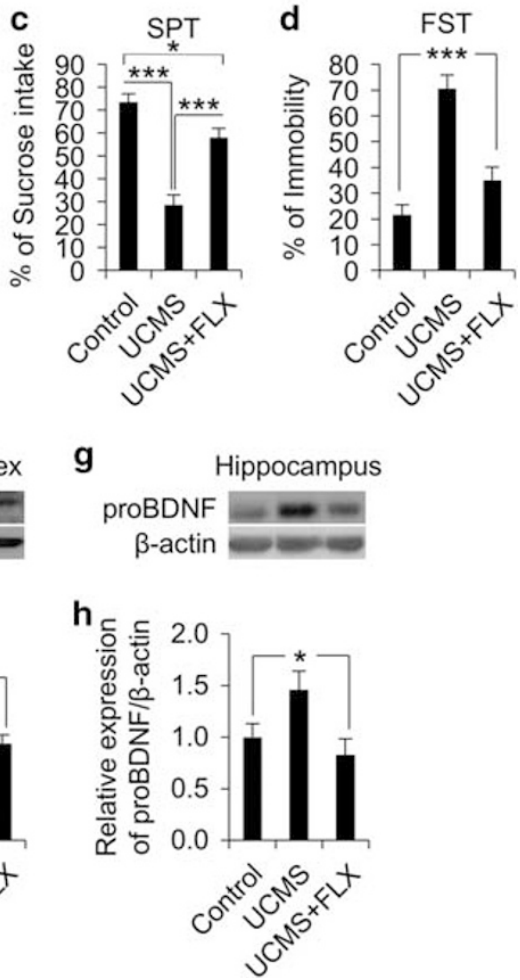

Figure I Chronic treatment with fluoxetine abolished UCMS-induced increase in proBDNF levels in mouse brains. (a) Timeline of fluoxetine administration, UCMS paradigm, and behavioral testing. (b-d) The percentages of immobility in open field test (b), sucrose intake in sucrose preference test (c), and immobility in forced swimming test (d) were compared among control, UCMS, and fluoxetine-treated UCMS (UCMS $+F L X)$ mice. $(e, g)$ The protein levels of proBDNF in the neocortex (e) and hippocampus (g) were compared among control, UCMS, and UCMS+FLX mice; $\beta$-actin was detected as loading control. (f, h) Relative expression of proBDNF/ $\beta$-actin in both neocortex ( $f$ ) and hippocampus ( $h$ ) were quantified by densitometric analysis. FLX, fluoxetine; UCMS, unpredictable chronic mild stress; OFT, open field test; FST, forced swimming test; SPT, sucrose preference test; $n=12$ in each group, ${ }^{*} p<0.05$, *** $p<0.0$ I, **** $p<0.00$ I (one-way ANOVA). All data are presented as mean \pm SEM.

ANOVA, $\mathrm{F}_{(2,33)}=27.67, p=0.000 ; p_{\text {(Control } v s \text { UCMS })}<0.001$, $p_{\text {(Control } v s \text { UCMS-FLX) }}=0.930, p_{\text {(UCMS } v s \text { UCMS-FLX })}<0.001$, LSD; Figure 1b). The percentage of sucrose intake in the sucrose preference test was significantly decreased in UCMS mice $v s$ control mice and fluoxetine was able to reverse this behavioral impairment in the UCMS mice; no significant difference was found between control and fluoxetine-treated UCMS mice (one-way ANOVA, $\mathrm{F}_{(2,33)}=31.03, p<0.001$; $P_{\text {(Control }} v s \quad$ UCMS) $<0.001, \quad P_{\text {(Control }}$ vs UCMS-FLX) $=0.012$, $p_{\text {(UCMS vs UCMS-FLX) }}<0.001$, LSD; Figure $1 c$ ). In addition, the percentage of immobility in the forced swimming test was increased in UCMS mice $v s$ control mice, and fluoxetine significantly reduced the immobility in these mice; no significant difference was found between control and fluoxetinetreated UCMS mice (one-way ANOVA, $\mathrm{F}_{(2,33)}=27.18$, 
a

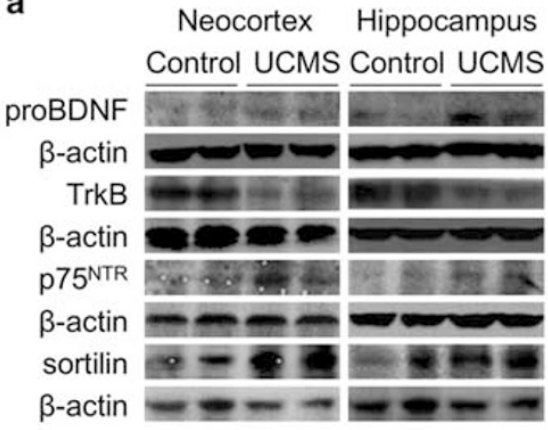

b
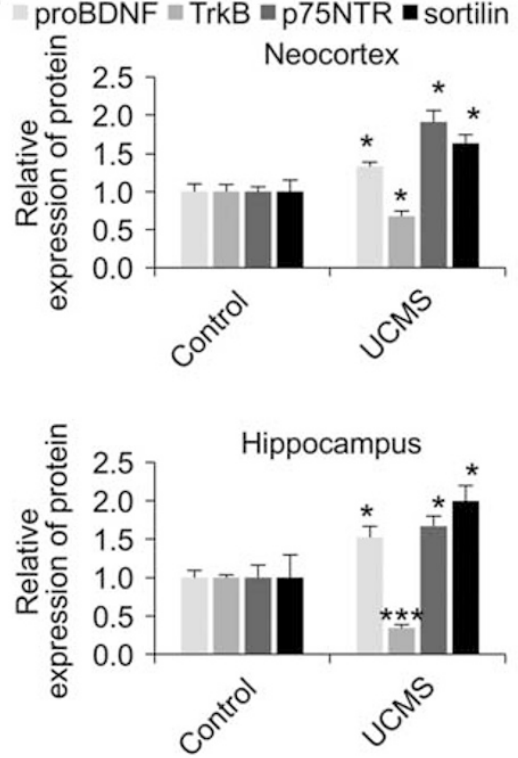

Neocortex Hippocampus Control UCMS Control UCMS

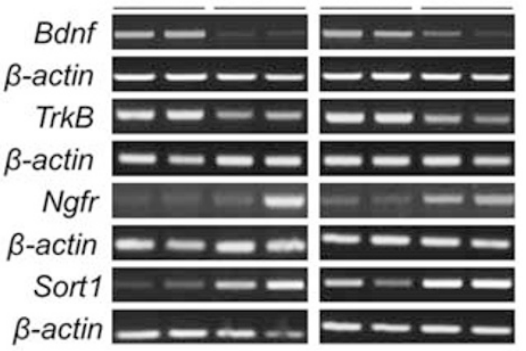

e $\quad B d n f=T r k B=N g f r=$ Sort1

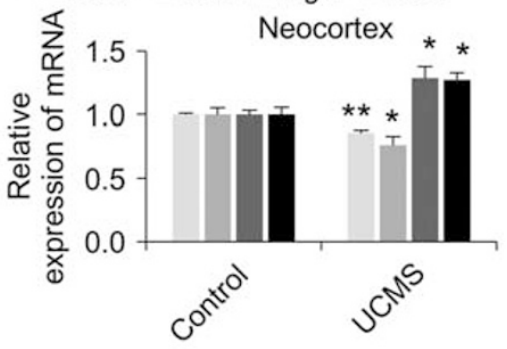

f

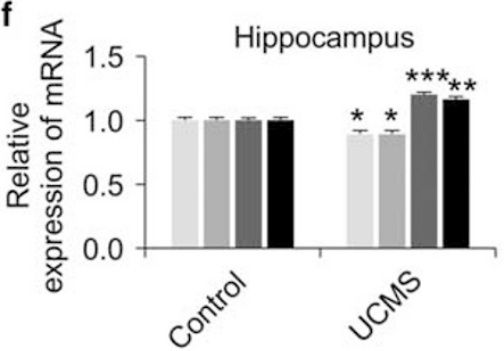

Figure 2 UCMS activated proBDNF/p75 NTR/sortilin and deactivated BDNF/TrkB signaling in rat brains. (a) The protein levels of proBDNF, TrkB, p $75^{N T R}$, and sortilin in the neocortex and hippocampus were compared between control and UCMS rats; $\beta$-actin was detected as loading control. (b, c) Relative expression of proBDNF/ $\beta$-actin, TrkB/ $\beta$-actin, $p 75^{N T R} / \beta$-actin, and sortilin/ $\beta$-actin in both neocortex (b) and hippocampus (c) were quantified by densitometric analysis. (d) The mRNA levels of Bdnf, TrkB, Ngfr, and Sortl in the neocortex and hippocampus were compared between control and UCMS rats; $\beta$-actin was detected as loading control. (e, f) Relative expression of Bdnf/ $\beta$-actin, TrkB/ $\beta$-actin, Ngfr/ $\beta$-actin, and Sort / $/ \beta$-actin in both neocortex (e) and hippocampus (f) were quantified by densitometric analysis. UCMS, unpredictable chronic mild stress; $n=5$ in each group, $* p<0.05$, *** $p<0.0$ I, **** $p<0.00$ I (Student's $t$-test). All data are presented as mean \pm SEM.

$p<0.001 ; p_{\text {(Control } v s \text { UCMS })}<0.001, p_{\text {(Control } v s \text { UCMS-FLX) }}=0.057$, $p_{\text {(UCMS vs UCMS-FLX) }}<0.001$, LSD; Figure $1 \mathrm{~d}$ ). Western blot analysis of proBDNF levels in the brain showed that the levels of proBDNF in both neocortex (one-way ANOVA, $\mathrm{F}_{(2,15)}=10.23$, $p=0.003 ; p_{\text {(Control } v s \text { UCMS })}=0.002, p_{\text {(Control } v s \text { UCMS-FLX })}=0.912$, $p_{\text {(UCMS vs UCMS-FLX) }}=0.003$, LSD; Figures $1 \mathrm{e}$ and $\mathrm{f}$ ) and hippocampus (one-way ANOVA, $\mathrm{F}_{(2,15)}=4.35, \quad p=0.032$; $p_{\text {(Control vs UCMS })}=0.045, p_{\text {(Control vs }}$ UCMS-FLX) $=0.449$, $p_{\text {(UCMS vs UCMS-FLX) }}=0.012$, LSD; Figures $1 \mathrm{~g}$ and $\mathrm{h}$ ) were increased after UCMS procedure and this increase was abolished by fluoxetine treatment.

\section{UCMS Caused Behavioral Impairments and Increased proBDNF/p $75^{\mathrm{NTR}} /$ Sortilin Signaling in Rats}

As it is well known that the BDNF/TrkB signaling is inhibited in the brains of stressed rodents and depressed patients, we then asked a question of whether proBDNF/ $\mathrm{p} 75^{\mathrm{NTR}} /$ sortilin signaling is activated in the brain of rats under stress. To address this question, we used a group of stressed rats $(n=10)$ exposed to the 21-day UCMS protocol along with a nonstressed group as control $(n=10)$ (Supplementary Figure S3A). Open field (day 22), forced swimming (day 23), and sucrose preference (day 24) tests were performed at the end of the UCMS procedure. The UCMS rats showed a significantly shorter traveling distance (Student's $t$-test, $t_{(18)}=5.77, p<0.001$; Supplementary Figure S3B), increased percentage of immobility (Student's $t$-test, $t_{(18)}=-16.52, p<0.001$; Supplementary Figure S3C), and decreased rearing numbers (Student's $t$-test, $t_{(18)}=10.48$, $p<0.001$, Supplementary Figure S3D) in the open field test compared with control rats. Moreover, the UCMS rats also showed a decreased percentage of sucrose intake in the sucrose preference test (Student's $t$-test, $t_{(18)}=14.38$, $p<0.001$, Supplementary Figure S3E) and an increased percentage of immobility (Student's $t$-test, $t_{(18)}=-12.93$, $p<0.001$, Supplementary Figure S3F) in the forced 
swimming test relative to control rats. Neocortical and hippocampal tissues were isolated and the changes in pro $\mathrm{BDNF} / \mathrm{p} 75^{\mathrm{NTR}} /$ sortilin and $\mathrm{BDNF} / \mathrm{TrkB}$ signaling were detected by western blot and RT-PCR analyses. Western blotting results showed that both cortical (Student's $t$-test, proBDNF, $t_{(8)}=-7.77, p=0.029$; TrkB, $t_{(8)}=8.35, p=0.033$, p $75^{\mathrm{NTR}}, \quad t_{(8)}=-9.05, \quad p=0.042 ; \quad$ sortilin, $t_{(8)}=-9.95$, $p=0.039$, Figures $2 \mathrm{a}$ and $\mathrm{b}$ ) and hippocampal (Student's $t$-test, proBDNF, $t_{(8)}=10.06, p=0.013 ; \operatorname{TrkB}, t_{(8)}=25.54$, $p=0.001, \mathrm{p} 75^{\mathrm{NTR}}, t_{(8)}=-4.57, p=0.024$; sortilin, $t_{(8)}=5.20$, $p=0.038$, Figures $2 \mathrm{a}$ and $\mathrm{c}$ ) protein levels of proBDNF, p $75^{\mathrm{NTR}}$, and sortilin were significantly increased but TrkB levels were decreased in UCMS rats vs control rats. Furthermore, RT-PCR results showed significantly decreased mRNA levels of Bdnf and TrkB but increased levels of $N g f r$ (the gene of $\mathrm{p} 75^{\mathrm{NTR}}$ ) and Sort1 (the gene of sortilin) in both neocortex (Student's t-test, $B d n f, t_{(8)}=5.07, \quad p=0.007$; $\operatorname{TrkB}, t_{(8)}=3.14, \quad p=0.035, \quad N g f r, t_{(8)}=-2.54, p=0.044$; Sort1, $t_{(8)}=-3.38, \quad p=0.028$, Figures $2 \mathrm{~d}$ and e) and hippocampus (Student's $t$-test, $B d n f, t_{(8)}=4.24, p=0.012$; $\operatorname{TrkB}, t_{(8)}=4.20, p=0.042, \mathrm{Ngfr}, t_{(8)}=-4.34, p=0.005$; Sort 1 , $t_{(8)}=-3.93, p=0.008$, Figures $2 \mathrm{~d}$ and f) of UCMS rats $v s$ control rats.

\section{Anti-proBDNF Antibody Improves UCMS-Induced Impairments of Mood-Related Behaviors}

The above results have shown that chronic stress induced depression-like behavior and activated proBDNF-mediated p $75^{\mathrm{NTR}} /$ sortilin cell signaling in rats; we therefore asked a question of whether blocking proBDNF could change the stress-induced mood abnormalities. We employed four groups of rats ( $n=6-11$ each) exposed to the 21-day UCMS protocol and injected with anti-proBDNF antibodies through i.c.v. and i.p. approaches in two of the UCMS groups; meanwhile, the same volumes of normal sheep serum (IgG) were injected into the other two UCMS groups (Figure 3a). Afterwards, behavioral tests were performed to assess the effect of anti-proBDNF antibody on mood-related behaviors. UCMS rats treated with anti-proBDNF through both i.c.v. and i.p. approaches showed a significant increase in the traveling distance (Student's $t$-test, i.c.v., $t_{(20)}=6.82$, $p<0.001$; i.p., $t_{(10)}=5.65, p<0.001$; Figure $3 b$ ), a decrease in the percentage of immobility (Student's $t$-test, i.c.v., $t_{(20)}=-11.08, \quad p<0.001 ; \quad$ i.p., $\quad t_{(10)}=-10.07, \quad p<0.001$; Figure $3 \mathrm{c}$ ), and increased rearing numbers (Student's $t$-test, i.c.v., $\quad t_{(20)}=6.68, \quad p<0.001$; i.p., $t_{(10)}=9.09, \quad p<0.001$; Figure $3 \mathrm{~d}$ ) in the open field test compared with the UCMS rats that received normal IgG. Moreover, sucrose preference test showed an increased percentage of sucrose intake (Student's $t$-test, i.c.v., $\quad t_{(20)}=10.67, \quad p<0.001$; i.p., $t_{(10)}=9.03, p<0.001$; Figure $3 \mathrm{e}$ ), whereas forced swimming test showed a decreased percentage of immobility (Student's $t$-test, i.c.v., $t_{(20)}=-10.09, p=0.023$; i.p., $t_{(10)}=-9.85$, $p<0.001$; Figure $3 \mathrm{f}$ ) in UCMS rats treated with i.c.v. and i.p. anti-proBDNF injections relative to UCMS rats treated with IgG. In addition, a cross-section image of normal rat brain was presented that showed the validity of i.c.v. microinjection using $1 \times$ SDS-PAGE sample loading buffer (Figure 3g).
Overexpression of proBDNF Did Not Exacerbate whereas Overexpression of BDNF Improved the UCMS-Induced Mood-Related Behaviors

In this set of experiments, we wished to investigate the effects of overexpression of proBDNF $v s$ mature BDNF on behavior in rats exposed to the 21-day UCMS protocol. We hypothesized that proBDNF overexpression will exacerbate the UCMS-induced depression-like behaviors in rats, whereas BDNF overexpression will improve such behaviors. To test this hypothesis, we employed another three groups ( $n=7$ each) of UCMS rats and injected them with viral vectors AAV-proBDNF, AAV-BDNF, and AAV-EGFP (as a control) in the tibialis anterior muscle, respectively (Figure 4a). After 2 weeks, behavioral tests were performed to assess the effect of the treatments on mood-related behaviors. UCMS rats treated with AAV-BDNF displayed a significant increase in the traveling distance (one-way ANOVA, $\mathrm{F}_{(2,18)}=73.62, \quad p<0.001$; $p_{\text {(AAV-EGFP } v s \text { AAV-BDNF })}<0.001, p_{\text {(AAV-EGFP } v s \text { AAV-proBDNF })}=$ $0.274, p_{\text {(AAV-BDNF } v s \text { AAV-proBDnF) }}<0.001$, LSD; Figure $\left.4 \mathrm{~b}\right)$ and rearing numbers ${ }^{v s}$ (one-way ANOVA, $\mathrm{F}_{(2,18)}=63.64$, $p<0.001 ; p_{\text {(AAV-EGFP }} v s$ AAV-BDNF) $<0.001, p_{\text {(AAV-EGFP }} v s$ AAV-proBDNF $)=0.030, \quad p_{(\mathrm{AAV}-\mathrm{BDNF}} \quad v s$ AAV-proBDNF $)<0.001$, LSD; Figure 4d), but a decrease in the percentage of immobility (one-way ANOVA, $\mathrm{F}_{(2,18)}=73.62, p<0.001$; $p_{\text {(AAV-EGFP } v s \text { AAV-BDNF })}<0.001, p_{\text {(AAV-EGFP } v s \text { AAV-proBDNF })}=$ $0.274, p_{\text {(AAv-BDNF vs AAv-proBDnF) }}<0.001$, LSD; Figure $4 \mathrm{c}$ ), in the open field test when compared with UCMS rats treated with AAV-EGFP or AAV-proBDNF. There was no significant difference in behavioral outcomes between UCMS rats receiving AAV-proBDNF and AAV-EGFP. Moreover, sucrose preference test showed that the percentage of sucrose intake was increased in UCMS rats treated with AAV-BDNF relative to UCMS rats treated with AAV-proBDNF or AAV-EGFP, whereas no difference was found between UCMS rats treated with AAV-EGFP vs $\mathrm{AAV}$-proBDNF (one-way ANOVA, $\mathrm{F}_{(2,18)}=28.92, p<0.001$; $\left.p_{(\mathrm{AAV}-\mathrm{EGFP}} v s \mathrm{AAV}-\mathrm{BDNF}\right)=0.030, p_{(\mathrm{AAV}-\mathrm{EGFP}} v s \mathrm{AAV}$-proBDNF $)=$ $0.530, p_{(\mathrm{AAY}-\mathrm{BDNF}} v{ }_{\mathrm{AAV}-\mathrm{proBDNF})}=0.032, \mathrm{LSD} ;$ Figure $\left.4 \mathrm{e}\right)$. In addition, forced swimming test showed a significant decrease in the percentage of immobility in UCMS rats injected with AAV-BDNF vs AAV-EGFP or $A A V$-proBDNF, whereas no difference was found between UCMS rats injected with AAV-EGFP vs AAV-proBDNF (one-way ANOVA, $\mathrm{F}_{(2,18)}=62.38, p<0.001 ; p_{\text {(AAV-EGFP }} v s$

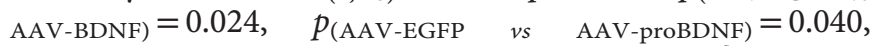
$p_{\text {(AAV-BDNF } v \text { AAV-proBDNF) }}<0.001$, LSD; Figure $4 \mathrm{f}$ ).

\section{Anti-proBDNF and AAV-BDNF Reversed the UCMS-Elicited Decrease of Dendritic Spines, but AAV-proBDNF Did Not Show Any Effect}

Given that anti-proBDNF and AAV-BDNF treatments have alleviated the UCMS-induced mood abnormalities whereas AAV-proBDNF treatment has not exhibited any effect, we further tested their underlying mechanisms in regulation of mood-related behavior. As decreased spine length has been observed in chronically stressed rodents (Chen et al, 2010; Li et al, 2011; Radley et al, 2006), we performed Golgi staining on brain sections from the above groups of rats presented in Supplementary Figure S3. 
a
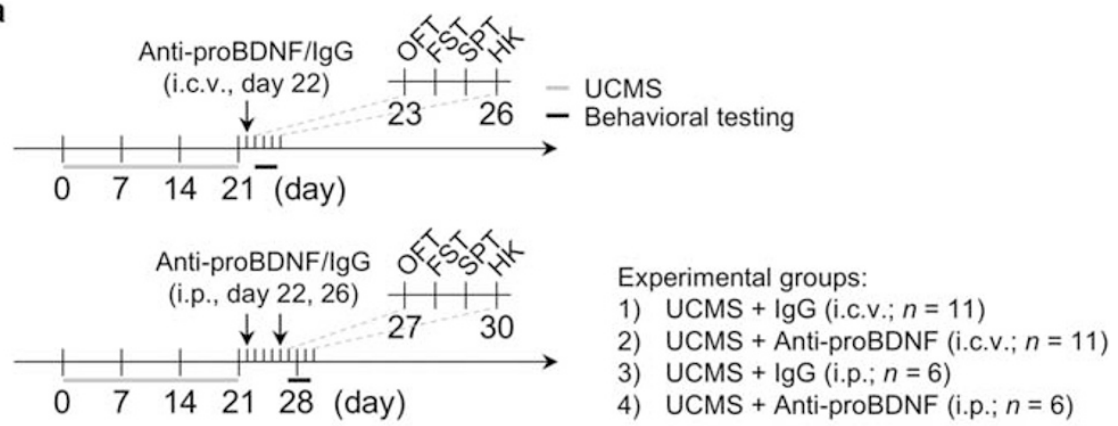

4) UCMS + Anti-proBDNF (i.p.; $n=6$ )

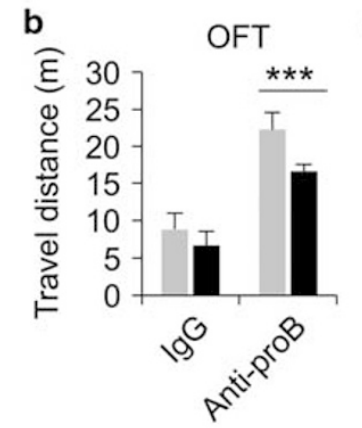

C OFT
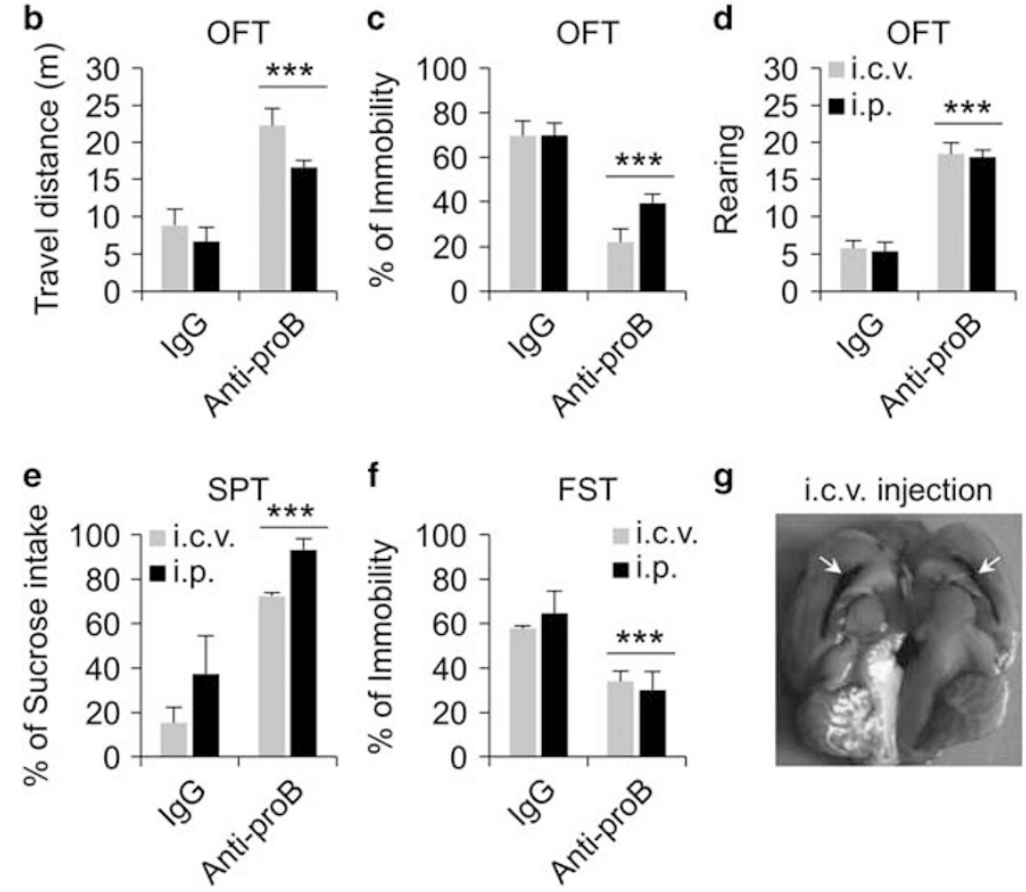

g

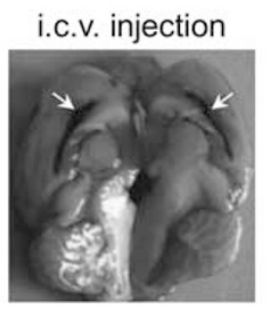

Figure 3 Anti-proBDNF antibody improves UCMS-induced depression-like behaviors in rats. (a) Timeline of UCMS paradigm, antibody injection and behavioral testing. (b-f) The travel distances (b), percentage of immobility (c), and rearing numbers (d) in open field test, the percentages of sucrose intake in sucrose preference test (e), as well as immobility in forced swimming test ( $f$ ) were compared between UCMS rats that were i.c.v. or i.p. injected with normal sheep serum and anti-proBDNF antibody (i.c.v., $20 \mu \mathrm{g}$, I $\mu \mathrm{g} / \mu \mathrm{l}$ antibody; i.p., $10 \mu \mathrm{l}$ per g of body weight, $0.08 \mu \mathrm{g} / \mu \mathrm{l}$ antibody). (g) The validation of i.c.V. microinjection with I $\times$ SDS-PAGE sample loading buffer. UCMS, unpredictable chronic mild stress; OFT, open field test; FST, forced swimming test; SPT, sucrose preference test; HK, humane killing; i.c.V., intraventricular; i.p., intraperitoneal; Anti-proB, anti-proBDNF; $n=6-1$ I in each group, **** $p<0.00$ I (Student's t-test). All data are presented as mean \pm SEM.

The results showed that UCMS significantly decreased the spine length of both cortical (Student's $t$-test, $t_{(18)}=14.17$, $p<0.001$; Figures $5 \mathrm{a}$ and $\mathrm{b}$ ) and hippocampal (Student's $t$-test, $t_{(18)}=16.63, p<0.001$; Figures $5 \mathrm{a}$ and $\left.\mathrm{b}\right)$ neurons compared with nonstressed control group. UCMS rats treated with anti-proBDNF via both i.c.v. and i.p. showed significant increases in the spine length in both cortical (Student's $t$-test, i.c.v., $t_{(20)}=9.03, p<0.001$; i.p., $t_{(10)}=5.00$, $p=0.001$; Figures $5 \mathrm{a}$ and $c$ ) and hippocampal (Student's $t$-test, i.c.v., $t_{(20)}=10.06, p<0.001$; i.p., $t_{(10)}=4.77, p=0.001$; Figures $5 \mathrm{a}$ and $\mathrm{d}$ ) neurons compared with UCMS rats injected with normal IgG. Moreover, UCMS rats with AAV-BDNF treatment also showed significant increases in the spine length in both cortical (one-way ANOVA, $\mathrm{F}_{(2,18)}=214.20, p<0.001 ; p$ (AAV-EGFP vs AAV-BDNF) $<0.001$, $p($ AAV-EGFP $\quad v s \quad$ AAV-proBDNF $)=0.448, \quad p_{(\mathrm{AAV}-\mathrm{BDNF}} \quad v s$ AAV-
proBDNF) $<0.001$, LSD; Figure 5e) and hippocampal (oneway ANOVA, $\mathrm{F}_{(2,18)}=205.00, p<0.001$; $p_{(\text {AAV-EGFP }} v s$ AAVBDNF $)<0.001, p_{(\text {AAV-EGFP } v s \text { AAV-proBDNF })}=0.034, p_{(\text {AAV-BDNF }}$ vs AAV-proBDNF) $<0.001$, LSD; Figure $5 \mathrm{f}$ ) neurons compared with UCMS rats treated with AAV-EGFP or AAV-proBDNF, whereas there was no significant difference in the spine length in UCMS rats injected with AAV-proBDNF vs normal IgG.

\section{Brain Injection of AAV-proBDNF Increased Depression-Like Behavior But Did Not Influence Anxiety-Like Behavior or Spontaneous Locomotor Activity in Naive Rats}

We have found that i.m. injection of AAV-proBDNF did not significantly change the mood-related phenotypes in UCMS animals, and this could possibly be due to a ceiling effect 
a

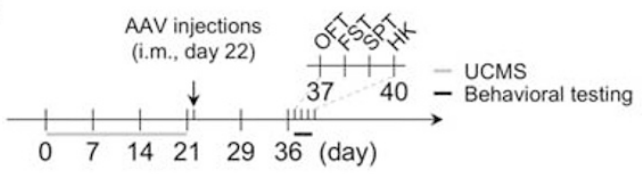

Experimental groups:

1) UCMS + AAV-EGFP (i.m.; $n=7$ )

2) UCMS + AAV-BDNF (i.m.; $n=7)$

3) UCMS + AAV-proBDNF (i.m.; $n=7)$
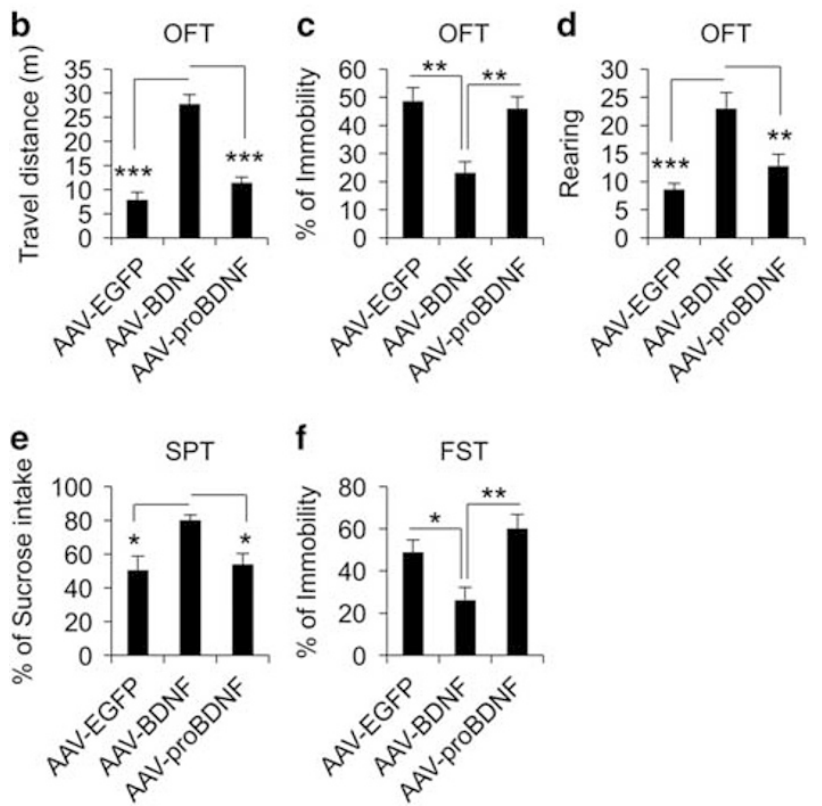

Figure 4 Overexpression of proBDNF did not exacerbate the UCMS-induced depression-like behaviors in rats. (a) Timeline of UCMS paradigm, injection of AAV vector, and behavioral testing. (b-f) The travel distances (b), percentage of immobility (c), and rearing numbers (d) in open field test, the percentages of sucrose intake in sucrose preference test (e), as well as immobility in forced swimming test $(f)$ were compared among UCMS rats that were i.m. injected with AAV-EGFP, AAV-BDNF, and AAVproBDNF $\left(50 \mu \mathrm{l}, 0.5 \times 10^{9} \mathrm{VG} / \mu \mathrm{l}\right) . \mathrm{UCMS}$, unpredictable chronic mild stress; OFT, open field test; FST, forced swimming test; SPT, sucrose preference test; HK, humane killing; i.m., intramuscular; $n=7$ in each group, $* p<0.05$, **** $p<0.0$ I, ***** $p<0.00$ I (one-way ANOVA). All data are presented as mean \pm SEM.

when proBDNF levels in the brain become too high. To test this hypothesis, we injected AAV-proBDNF in the lateral ventricles of normal naive rats that did not undergo the UCMS protocol (AAV-EGFP was injected as control) and examined the spontaneous locomotor activity and anxietylike and depression-like behaviors before and after injection (as shown in Figure 6a). It was found that i.c.v. injection of $\mathrm{AAV}$-proBDNF did not change locomotor activity of rats as evidenced by no significant difference in travel distance (two-way ANOVA, injection factor: $\mathrm{F}_{(1,10)}=6.21, p=0.022$; proBDNF factor: $\mathrm{F}_{(1,10)}=0.56, \quad p=0.463$; injection $\times$ proBDNF: $\mathrm{F}_{(1,10)}=0.39, p=0.539$; Figure $6 \mathrm{~b}$ ) or percentage of immobility (two-way ANOVA, injection factor: $\mathrm{F}_{(1,10)}=8.64, p=0.008 ;$ proBDNF factor: $\mathrm{F}_{(1,10)}=0.20$, $p=0.659 ; \quad$ injection $\times$ proBDNF: $\quad \mathrm{F}_{(1,10)}=0.25, \quad p=0.623$; Figure $6 \mathrm{c}$ ) in the open field test between AAV-EGFP and AAV-proBDNF groups. Besides, AAV-proBDNF did not alter anxiety-like behavior in rats as evidenced by no significant difference in the percentage of time spent in central zone (two-way ANOVA, injection factor: $\mathrm{F}_{(1,10)}=2.04, \quad p=0.169 ;$ proBDNF factor: $\mathrm{F}_{(1,10)}=0.16$, $p=0.697 ; \quad$ injection $\times$ proBDNF: $\quad \mathrm{F}_{(1,10)}=0.27, \quad p=0.612$; Figure 6d) in the open field test. However, AAV-proBDNF increased the depression-like behavior as indicated by a significant increase in the percentage of immobility in the forced swimming test (two-way ANOVA, injection factor: $\mathrm{F}_{(1,10)}=0.38, p=0.544 ;$ proBDNF factor: $\mathrm{F}_{(1,10)}=5.58$, $p=0.028 ; \quad$ injection $\times$ proBDNF: $\quad \mathrm{F}_{(1,10)}=2.62, \quad p=0.121$; Figure 6e). To check the efficacy of AAV-proBDNF treatment to increase proBDNF levels in the brain, we performed an ELISA assay as previously described (Lim et al, 2015) and found that proBDNF levels were significantly increased in the neocortex (Student's $t$-test, $t_{(10)}=-13.54$, $p<0.001$; Figure 6f) and cerebellum (Student's $t$-test, $t_{(10)}=-2.81, p=0.019$; Figure 6f) but not in hippocampus (Student's $t$-test, $t_{(10)}=-1.69, p=0.121$; Figure $6 \mathrm{f}$ ). Histological examination showed that green fluorescence was detected in the brains of rats that received AAV-EGFP but not AAV-proBDNF, and a significant upregulation of proBDNF expression was detected in the brains of rats that received AAV-proBDNF but not AAV-EGFP (Figure 6g).

\section{DISCUSSION}

The present study investigated the role of proBDNF in regulation of mood-related behaviors. Our results showed that chronic stress leads to increased levels of proBDNF, p $75^{\mathrm{NTR}}$, and sortilin but decreased levels of TrkB in the neocortex and hippocampus in rodent brains. Injection of anti-proBDNF antibodies via i.c.v. and i.p. approaches both attenuated the chronic stress-induced decrease of exploratory and depression-like behaviors as well as the loss of dendritic spines. In addition, injection of AAV-BDNF via i.m. approach restored the abnormal behaviors and increased the dendritic spine growth caused by chronic stress, but AAV-proBDNF injection did not change the chronic stressinduced impairments in mood-related behaviors or spine morphology. The i.c.v. injection of AAV-proBDNF in naive rats resulted in depression-like behavior but not the other mood-related activities.

We first showed that mice exposed to the 28-day UCMS paradigm increased proBDNF levels in the neocortex and hippocampus, consistent with a recent study reporting that the proBDNF levels are increased in the neocortex of rats exposed to learned helplessness paradigm (Shirayama et al, 2015). Furthermore, we have previously found that the level of proBDNF in the serum and lymphocytes is increased in the patients with MDD (Zhou et al, 2013), suggesting that proBDNF is also dysregulated in MDD. The above findings may suggest that the upregulation of proBDNF is associated with the development and progression of mood disorders.

To further confirm the link between proBDNF and mood regulation, we tested whether antidepressant treatment can change the proBDNF levels in the brain. Our data showed that the first-line antidepressant drug fluoxetine, a serotonin reuptake inhibitor (Wong et al, 1974), reversed the UCMSinduced depression-like behavior and upregulation of proBDNF in the neocortex and hippocampus of mouse brains. These results are consistent with previous studies reporting that chronic antidepressant treatments including 
a
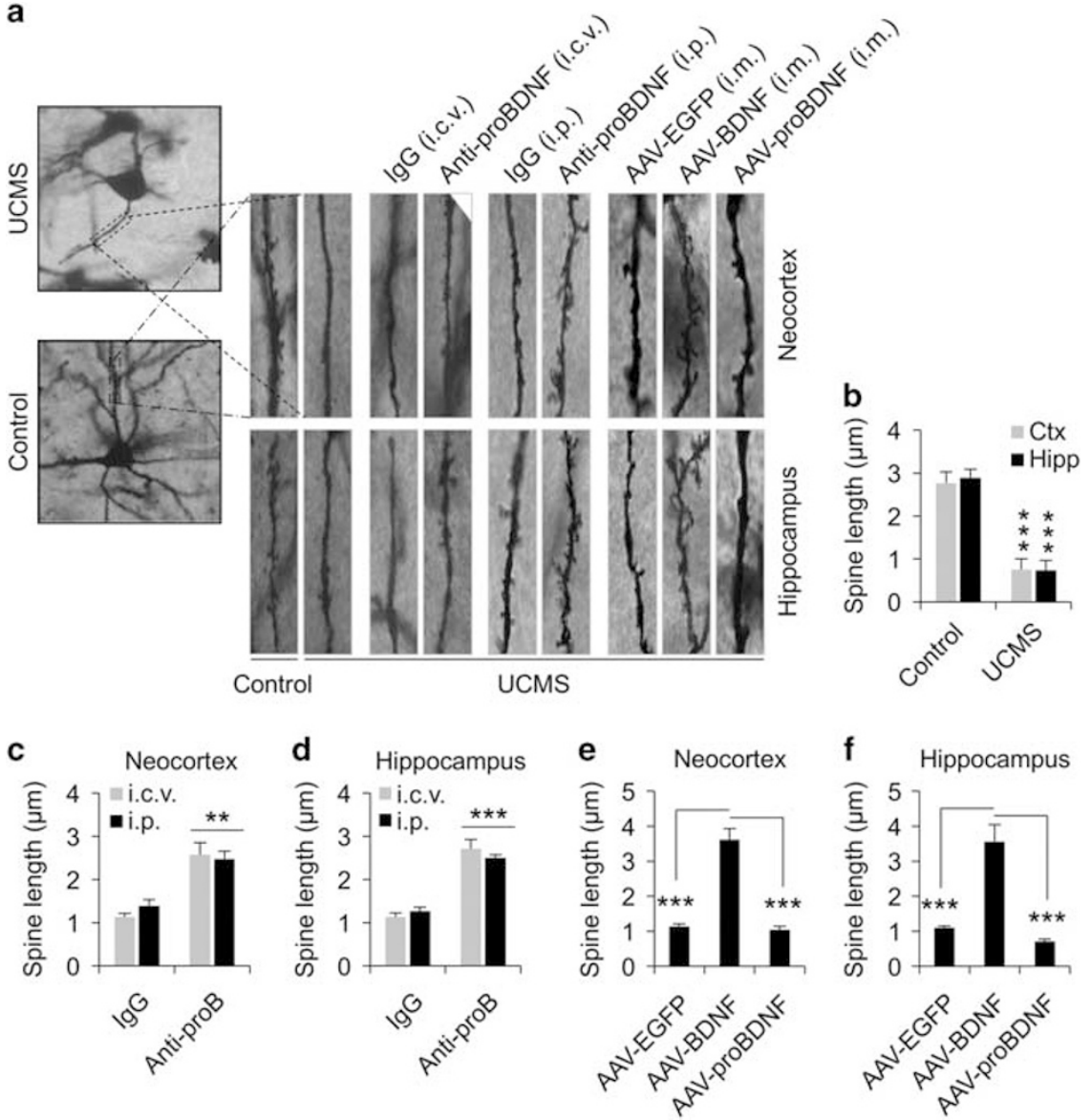

Figure 5 Anti-proBDNF antibody improved but AAV-proBDNF did not exacerbate the UCMS-induced reductions in the dendritic spine length in rats. (a) Representative images of dendritic spines in cortical and hippocampal neurons were compared among control, UCMS, UCMS treated with normal sheep IgG and anti-proBDNF (i.c.v.), UCMS with IgG and anti-proBDNF (i.p.), as well as UCMS with AAV-EGFP, AAV-BDNF, and AAV-proBDNF (i.m.). (b-f) The spine lengths of cortical and hippocampal neurons were quantified and compared between/among control and UCMS rats (b), IgG and anti-proBDNF (i.c.v. or i.p.) injected UCMS rats (c, d), and AAV-EGFP, AAV-BDNF, and AAV-proBDNF (i.m.) injected UCMS rats (e, f). UCMS, unpredictable chronic mild stress; i.c. V., intraventricular; i.p., intraperitoneal; i.m., intramuscular; AAV, adeno-associated virus; Ctx, neocortex; Hipp, hippocampus; $n=6-I I$ in each group, *** $p<0.01$, ***** $p<0.00$ I ( $b-d$, Student's $t$-test; e, f, one-way ANOVA). All data are presented as mean \pm SEM.

serotonin-selective reuptake inhibitors increased the $B d n f$ transcriptional levels in rodent brains (Nibuya et al, 1995, 1996). Our study provides the first evidence supporting a novel mechanism underlying the efficacy of fluoxetine in the treatment of depression, that is, to reduce the proBDNF levels in the brain of depressive subjects. The result also supports the link between BDNF and serotoninergic systems in mood regulation.

To provide evidence that proBDNF is involved in the pathogenesis of depression in the chronic stress model, we examined the expression of proBDNF-related signaling molecules. We found that proBDNF/p $75^{\mathrm{NTR}} /$ sortilin neuronal apoptotic signaling was activated in the brains of UCMS rats. However, the transcriptional level of Bdnf in both neocortex and hippocampus of rats with UCMS was reduced, consistent with the studies showing that both chronic and acute stress paradigms reduce the hippocampal Bdnf mRNA in animals (Duman and Monteggia, 2006; Smith et al, 1995; Vaidya et al, 1997). These findings suggest that stressinduced increase of proBDNF is not due to the increased $B d n f$ transcription but is likely because of the inhibition of proteolytic processing of proBDNF. Interestingly, a number of studies have shown that antidepressants facilitate the conversion of proBDNF to BDNF by upregulation of the proteolytic cleavage enzymes of proBDNF (Cao et al, 2014; Sartori et al, 2011; Segawa et al, 2013), supporting our hypothesis. Furthermore, chronic stress in the present study decreased both transcriptional and translational levels of TrkB in the neocortex and hippocampus of rats, consistent with the previous findings that $\operatorname{TrkB}$ mRNA expression is reduced in pituitary gland of rats exposed to immobilization stress (Givalois et al, 2001). Moreover, the expression of p $75^{\mathrm{NTR}}$ and sortilin, at both protein and mRNA levels, was increased after chronic stress in the rat brains. As BDNF activates $\operatorname{TrkB}$ to mediate cell survival whereas proBDNF activates $\mathrm{p} 75^{\mathrm{NTR}}$ and sortilin complex to mediate cell death (Lee et al, 2001; Nykjaer et al, 2004; Teng et al, 2005), upregulation of proBDNF, p $75^{\mathrm{NTR}}$, and sortilin along with downregulation of TrkB suggests that chronic stress may activate the proBDNF/p $75^{\mathrm{NTR}} /$ sortilin neurodegenerative signaling pathway and inhibit the BDNF/TrkB cell survival signaling pathway. These data also suggest that imbalance between the two opposing pathways may contribute to the 
a
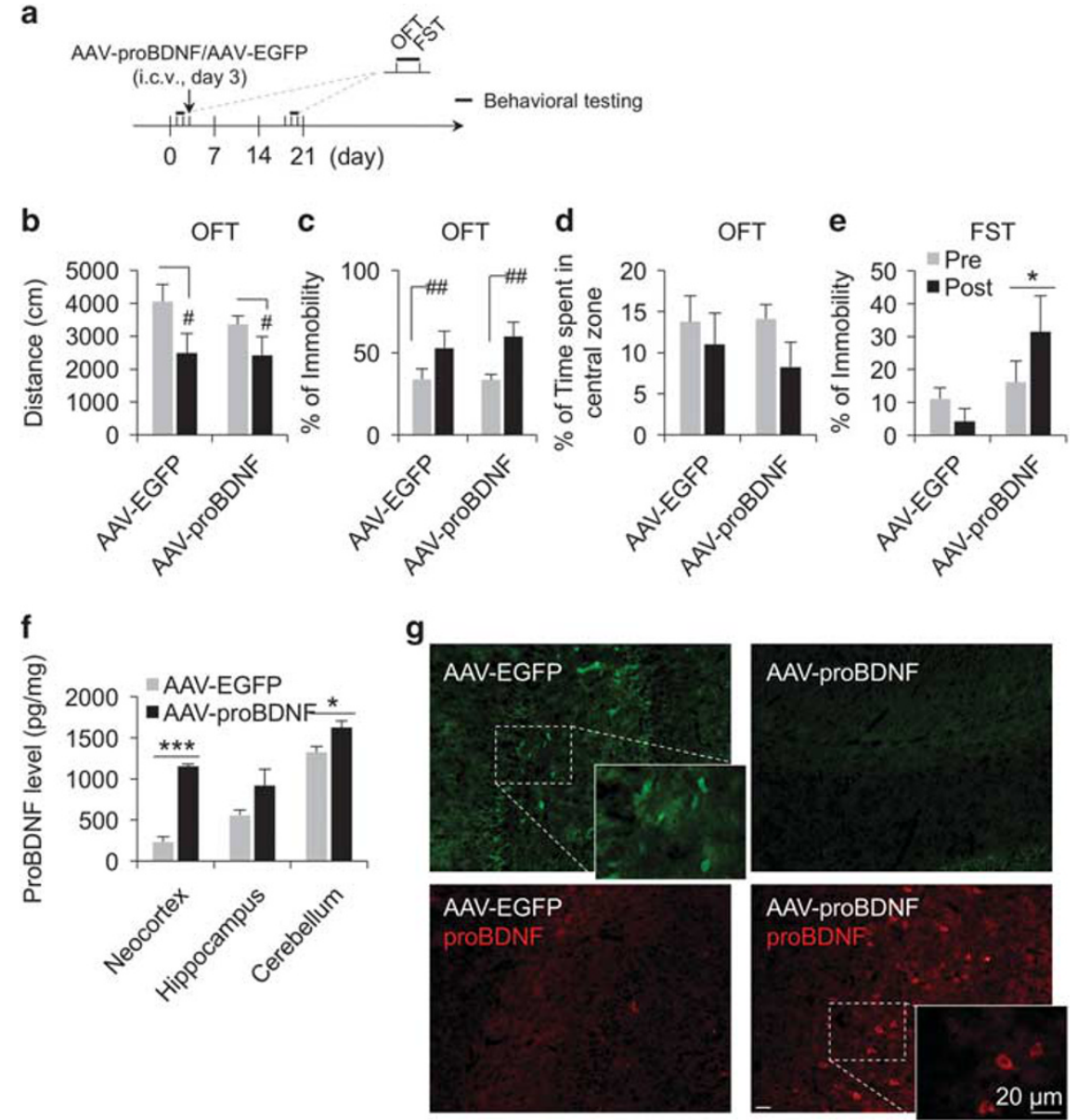

Figure 6 Overexpression of proBDNF in the brain increased depression-like behavior but not anxiety-like behavior in naive rats. (a) Timeline of injection of $A A V$ vector and behavioral testing. (b-e) The travel distances (b), percentage of immobility (c), and percentage of time spent in central zone (d) in the open field test, and the percentage of immobility in the forced swimming test (e) were compared at baseline (before injection) and after i.c.V. injection of AAV-EGFP or AAV-proBDNF (after injection). (f) The expression level of proBDNF in the neocortex, hippocampus, or cerebellum was detected by ELISA assay and compared between rats that received i.c.v. injection of AAV-EGFP or AAV-proBDNF. (g) The expression level of EGFP (direct imaging) or proBDNF (immunostaining using a specific antibody) in brain sections was compared between rats that received i.c.v. injection of AAV-EGFP or AAV-proBDNF; $n=6$ in each group; scale bar, $20 \mu \mathrm{m}$. OFT, open field test; i.c.v., intraventricular; FST, forced swimming test; $* p<0.05$, ${ }^{*} * * * 0.00$ I (proBDNF factor); ${ }^{*} p<0.05$, $\# \#<0.01$ (injection factor). (b-e, two-way ANOVA; f, Student's t-test). All data are presented as mean \pm SEM.

pathogenesis of major depression and neurodegeneration during stress.

Our previous study showed that the neurite outgrowth of dorsal root ganglion neurons in vitro is inhibited upon treatment with proBDNF, but enhanced upon treatment with anti-proBDNF (Sun et al, 2012). As this anti-proBDNF antibody can neutralize the biological activities of proBDNF in neurons (Fan et al, 2008; Sun et al, 2012; Xu et al, 2011), we wished to establish whether anti-proBDNF treatment can reverse the behavioral and pathological impairments in rats caused by chronic stress. The present study showed that i.c.v. or i.p. injection of anti-proBDNF antibodies in UCMS rats reversed the chronic stress-induced decrease of exploratory activity, depression-like behaviors, and spine loss, suggesting that both central and peripheral inhibition of proBDNF can attenuate the chronic stress-elicited mood dysregulation and brain damage. Previous studies have shown that central or peripheral infusion of BDNF produces anti-depressant effects in animal models (Schmidt and Duman et al, 2010; Shirayama et al, 2002; Siuciak et al, 1997). Here, we provide evidence that targeting central or peripheral proBDNF by using a specific proBDNF antibody can also be considered as antidepressant treatment option. In line with previous studies we also found that overexpression of mature BDNF using viral vector via i.m. injection significantly reversed the chronic stress-induced behavioral and pathological changes, but overexpression of proBDNF did not further exacerbate these effects. This result suggests that the endogenous level of proBDNF under the chronic stress conditions may reach the saturation level so that additional proBDNF may not generate additional effects. One of the limitations of the present study is the lack of the study to correlate depression scores with blood or proBDNF levels. Future studies using large samples may address this limitation by measuring $\mathrm{mBDNF} /$ proBDNF levels in the blood and brain after AAV-BDNF and AAV-proBDNF injections in stressed animals. However, our subsequent experiment conduced in naive rats (not exposed to chronic stress) showed that overexpression of proBDNF in the brain via AAV vector injection significantly increased the depression-like behavior 
but not anxiety-like behavior. These results further strengthen our hypothesis that proBDNF plays a detrimental role in mood regulation. On the contrary, overexpression of mature BDNF successfully improved the UCMS-caused damages, suggesting that the balance of mature BDNF to proBDNF is critical in the maintenance of brain homeostasis and prevents the development of depressive behaviors. It is highly possible that supplementation of mature BDNF counteracts the proBDNF/p $75^{\mathrm{NTR}} /$ sortilin signaling in depression and exerts its therapeutic effects as seen in the previous studies (Shirayama et al, 2002; Siuciak et al, 1997). Therefore, restoration of the balance between proBDNF and mature BDNF by inhibiting proBDNF or supplementing mature $\mathrm{BDNF}$ is more important than a simple upregulation of the BDNF gene in the treatment of depression.

In summary, this study provides evidence supporting a significant role of proBDNF in mood regulation. Our study suggests that proBDNF plays a detrimental role in depression in rodent models and can be a potential therapeutic target for the treatment of mood disorders.

\section{FUNDING AND DISCLOSURE}

The authors declare no conflict of interest.

\section{ACKNOWLEDGMENTS}

This research was supported by grants from Chinese MST 2011CB944200, NHMRC (APP1021408, APP1020567), and University of South Australia to X-FZ.

\section{REFERENCES}

Autry AE, Adachi M, Cheng P, Monteggia LM (2009). Genderspecific impact of brain-derived neurotrophic factor signaling on stress-induced depression-like behavior. Biol Psychiatry 66: 84-90.

Cao W, Duan J, Wang X, Zhong X, Hu Z, Huang F et al (2014). Early enriched environment induces an increased conversion of proBDNF to BDNF in the adult rat's hippocampus. Behav Brain Res 265: 76-83.

Chen Y, Rex CS, Rice CJ, Dubé CM, Gall CM, Lynch G et al (2010). Correlated memory defects and hippocampal dendritic spine loss after acute stress involve corticotropin-releasing hormone signaling. Proc Natl Acad Sci USA 107: 13123-13128.

Chourbaji S, Brandwein C, Gass P (2011). Altering BDNF expression by genetics and/or environment: impact for emotional and depression-like behaviour in laboratory mice. Neurosci Biobehav Rev 35: 599-611.

Chourbaji S, Hörtnagl H, Molteni R, Riva M, Gass P, Hellweg R (2012). The impact of environmental enrichment on sex-specific neurochemical circuitries-effects on brain-derived neurotrophic factor and the serotonergic system. Neuroscience 220: 267-276.

Duman RS, Monteggia LM (2006). A neurotrophic model for stressrelated mood disorders. Biol Psychiatry 59: 1116-1127.

Fan YJ, Wu LL, HY Li, Wang YJ, Zhou XF (2008). Differential effects of pro-BDNF on sensory neurons after sciatic nerve transection in neonatal rats. Eur J Neurosci 27: 2380-2390.

Givalois L, Marmigere F, Rage F, Ixart G, Arancibia S, Tapia-Arancibia L (2001). Immobilization stress rapidly and differentially modulates BDNF and TrkB mRNA expression in the pituitary gland of adult male rats. Neuroendocrinology 74: 148-159.
Huang EJ, Reichardt LF (2001). Neurotrophins: roles in neuronal development and function. Annu Rev Neurosci 24: 677.

Lang UE, Hellweg R, Gallinat J (2004). BDNF serum concentrations in healthy volunteers are associated with depression-related personality traits. Neuropsychopharmacology 29: 795-798.

Lee BH, Kim H, Park SH, Kim YK (2007). Decreased plasma BDNF level in depressive patients. J Affect Disord 101: 239-244.

Lee R, Kermani P, Teng KK, Hempstead BL (2001). Regulation of cell survival by secreted proneurotrophins. Science 294: 1945-1948.

Lewin GR, Barde Y-A (1996). Physiology of the neurotrophins. Annu Rev Neurosci 19: 289-317.

Li N, Liu R-J, Dwyer JM, Banasr M, Lee B, Son H et al (2011). Glutamate N-methyl-D-aspartate receptor antagonists rapidly reverse behavioral and synaptic deficits caused by chronic stress exposure. Biol Psychiatry 69: 754-761.

Lim Y, Zhong JH, Zhou XF (2015). Development of mature BDNF-specific sandwich ELISA. J Neurochem 134: 75-85.

Nibuya M, Morinobu S, Duman RS (1995). Regulation of BDNF and $\operatorname{trkB}$ mRNA in rat brain by chronic electroconvulsive seizure and antidepressant drug treatments. J Neurosci 15: 7539-7547.

Nibuya M, Nestler EJ, Duman RS (1996). Chronic antidepressant administration increases the expression of cAMP response element binding protein (CREB) in rat hippocampus. $J$ Neurosci 16: $2365-2372$.

Nykjaer A, Lee R, Teng KK, Jansen P, Madsen P, Nielsen MS et al (2004). Sortilin is essential for proNGF-induced neuronal cell death. Nature 427: 843-848.

Olff M, Langeland W, Gersons BP (2005). The psychobiology of PTSD: coping with trauma. Psychoneuroendocrinology 30: 974-982.

Piccinni A, Marazziti D, Catena M, Domenici L, Del Debbio A, Bianchi C et al (2008). Plasma and serum brain-derived neurotrophic factor (BDNF) in depressed patients during 1 year of antidepressant treatments. J Affect Disord 105: 279-283.

Radley JJ, Rocher AB, Miller M, Janssen WG, Liston C, Hof PR et al (2006). Repeated stress induces dendritic spine loss in the rat medial prefrontal cortex. Cerebral Cortex 16: 313-320.

Ruan C, Zhou F, He Z, Wang S, Yang C, Shen Y et al (2015). Mice deficient for wild-type p53-induced phosphatase 1 display elevated anxiety-and depression-like behaviors. Neuroscience 293: $12-22$.

Ruan CS, Wang SF, Shen YJ, Guo Y, Yang CR, Zhou FH et al (2014). Deletion of TRIM32 protects mice from anxiety-and depression-like behaviors under mild stress. Eur J Neurosci 40: 2680-2690.

Sartori C, Vieira A, Ferrari E, Langone F, Tongiorgi E, Parada C (2011). The antidepressive effect of the physical exercise correlates with increased levels of mature BDNF, and proBDNF proteolytic cleavage-related genes, $\mathrm{p} 11$ and tPA. Neuroscience 180: 9-18.

Schechter D (1998). Estrogen, progesterone, and mood. J Gend Specif Med 2: 29-36.

Schmidt HD, Duman RS (2010). Peripheral BDNF produces antidepressant-like effects in cellular and behavioral models. Neuropsychopharmacology 35: 2378-2391.

Segawa M, Morinobu S, Matsumoto T, Fuchikami M, Yamawaki S (2013). Electroconvulsive seizure, but not imipramine, rapidly up-regulates pro-BDNF and t-PA, leading to mature BDNF production, in the rat hippocampus. Int J Neuropsychopharmacol 16: $339-350$.

Sherwin BB (1991). The impact of different doses of estrogen and progestin on mood and sexual behavior in postmenopausal women. J Clin Endocrinol Metabol 72: 336-343.

Shirayama Y, AC-H Chen, Nakagawa S, Russell DS, Duman RS (2002). Brain-derived neurotrophic factor produces antidepressant effects in behavioral models of depression. J Neurosci 22: 3251-3261. 
Shirayama Y, Yang C, Zhang JC, Ren Q, Yao W, Hashimoto K (2015). Alterations in brain-derived neurotrophic factor (BDNF) and its precursor proBDNF in the brain regions of a learned helplessness rat model and the antidepressant effects of a TrkB agonist and antagonist. Eur Neuropsychopharmacol.

Siuciak JA, Lewis DR, Wiegand SJ, Lindsay RM (1997). Antidepressant-like effect of brain-derived neurotrophic factor (BDNF). Pharmacol Biochem Behav 56: 131-137.

Smith MA, Makino S, Kvetnansky R, Post RM (1995). Stress and glucocorticoids affect the expression of brain-derived neurotrophic factor and neurotrophin-3 mRNAs in the hippocampus. J Neurosci 15: 1768-1777.

Sun Y, Lim Y, Li F, Liu S, Lu JJ, Haberberger R et al (2012). ProBDNF collapses neurite outgrowth of primary neurons by activating RhoA. PLoS One 7: e35883.

Teng HK, Teng KK, Lee R, Wright S, Tevar S, Almeida RD et al (2005). ProBDNF induces neuronal apoptosis via activation of a receptor complex of p75NTR and sortilin. J Neurosci 25: 5455-5463.

Vaidya VA, Marek GJ, Aghajanian GK, Duman RS (1997). 5-HT2A receptor-mediated regulation of brain-derived neurotrophic factor mRNA in the hippocampus and the neocortex. $J$ Neurosci 17: 2785-2795.

Wong DT, Horng JS, Bymaster FP, Hauser KL, Molloy BB (1974). A selective inhibitor of serotonin uptake: Lilly 110140, 3-(ptrifluoromethylphenoxy)-N-methyl-3-phenylpropylamine. Life Sciences 15: 471-479.

Woo NH, Teng HK, Siao CJ, Chiaruttini C, Pang PT, Milner TA et al (2005). Activation of p75NTR by proBDNF facilitates hippocampal long-term depression. Nat Neurosci 8: 1069-1077.

Xu ZQ, Sun Y, Li HY, Lim Y, Zhong JH, Zhou XF (2011). Endogenous proBDNF is a negative regulator of migration of cerebellar granule cells in neonatal mice. Eur J Neurosci 33: 1376-1384.

Yang CR, Bai YY, Ruan CS, Zhou HF, Liu D, Wang XF et al (2015). Enhanced aggressive behaviour in a mouse model of depression. Neurotox Res 27: 129-142.

Yang CR, Zhang ZG, Bai YY, Zhou HF, Zhou L, Ruan CS et al (2014). Foraging activity is reduced in a mouse model of depression. Neurotox Res 25: 235-247.

Zhou L, Xiong J, Lim Y, Ruan Y, Huang C, Zhu Y et al (2013). Upregulation of blood proBDNF and its receptors in major depression. J Affect Disord 150: 776-784.

Supplementary Information accompanies the paper on the Neuropsychopharmacology website (http://www.nature.com/npp) 